$$
\text { "todd" — 2009/2/18 — 23:25 — page 303 — \#1 }
$$

\title{
Geometry Expressions: an interactive constraint based symbolic geometry system
}

\author{
PHILIP TODD
}

\begin{abstract}
Dynamic geometry systems such as Geometers' SketchPad or Cabri are productive environments for the exploration of geometric relationships. They are, however, strictly numeric, and this limits their applicability where the interplay between geometry and algebra are being studied. We present Geometry Expressions - a dynamic symbolic geometry environment. While retaining the ease of use of a typical dynamic geometry environment, Geometry Expressions diverges by using constraints rather than constructions as the primary geometry specification mechanism and by working symbolically rather than numerically. Constraints, such as distances and angles, are specified symbolically. Symbolic measurements for quantities such as distances, angles, areas, locus equations, are automatically computed by the system. We outline how these features combine to create a rich dynamic environment for exploring the interplay between geometry and algebra, between induction and proof.
\end{abstract}

Key words and phrases: symbolic geometry, geometry, algebra, CAS, constraints, educational software.

ZDM Subject Classification: R20.

\section{Introduction}

Dynamic Geometry software has been proven over the last twenty years to be a strong facilitator of discovery based learning [2], [3]. Meanwhile CAS has provided a means by which average students can carry out accurate algebraic manipulation. We present a new software application Geometry Expressions, which extends the geometry-centric discovery learning of the dynamic geometry

Copyright (C) 2008 by University of Debrecen 


$$
\text { "todd" — 2009/2/18 — 23:25 — page } 304-\# 2
$$

package into the symbol manipulation world of the computer algebra system, thus linking the twin themes of this conference.

Geometry Expressions, is a computer application that, unlike other dynamic geometry systems, can automatically generate algebraic expressions from geometric figures. For example, in Figure 1, the user has specified that the triangle is right and has short sides length $a$ and $b$. The system has calculated an expression for the length of the altitude in terms of $a$ and $b$.

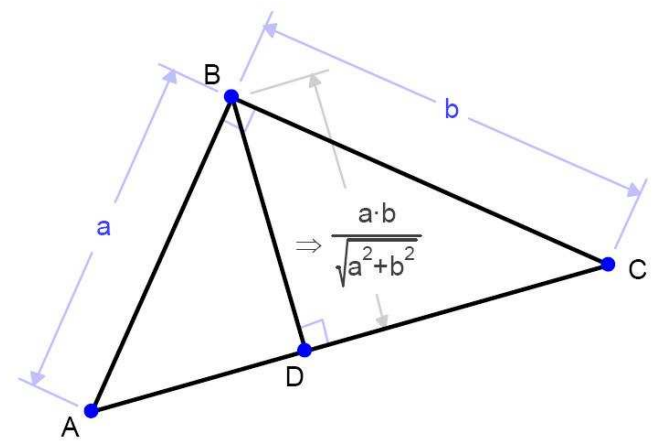

Figure 1. A Geometry Expressions model showing symbolic input and output

A demonstration version of Geometry Expressions may be downloaded from the web site www.geometryexpressions.com

Geometry Expressions is the fruition of a research effort on Symbolic Geometry at Saltire which dates back to a 1992-95 NSF funded research project. In the mid 90's there were two obstacles to the company directly productizing the technology:

1. It was too slow for problems of medium complexity on personal computers.

2. It relied on linking with an external algebra system (Mathematica or Maple), whereas it was desirable to have a product which could be used standalone.

In the intervening years, improvement to the symbolic geometry engine, along with the application of Moore's Law to hardware has removed the first obstacle: the system now works well for problems of medium complexity. Our internal benchmark of a medium complexity example is the side length of the equilateral triangle produced by Napoleon's theorem, in terms of the side lengths of the original triangle (Figure 2). The simplified expression in the figure is derived by Geometry Expressions in 2.5 seconds on a $2 \mathrm{GHz}$ Pentium PC running MS Windows XP. 


$$
\text { "todd" — 2009/2/18 — 23:25 — page 305 — \#3 }
$$

The second obstacle was removed by embedding a simple algebra system directly into Geometry Expressions. Generation and simplification of expressions along with simple algebraic manipulations are handled inside Geometry Expressions. If the user wants to do more sophisticated algebraic manipulation, he can cut and paste into a CAS (Geometry Expressions supports MathML in addition to proprietary formats for the leading algebra systems).

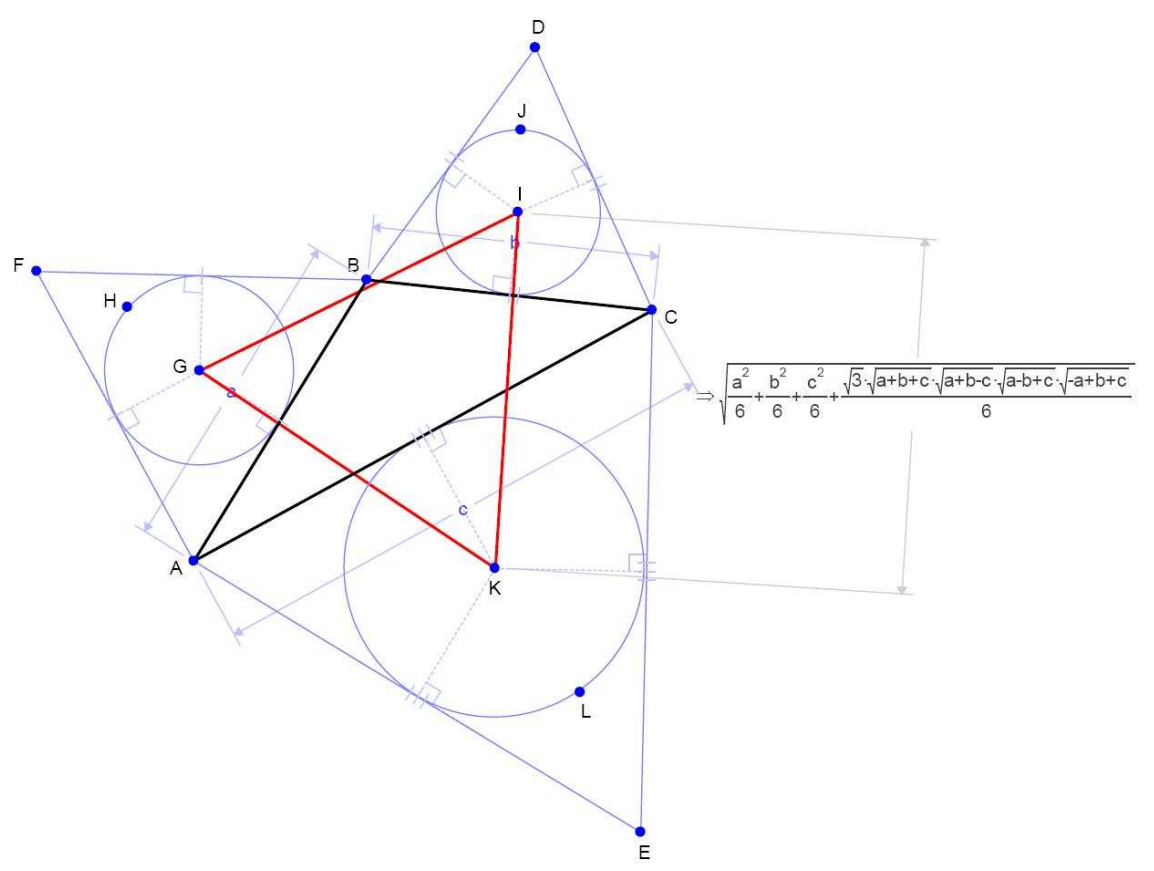

Figure 2. Length of triangle side in Napoleon's Theorem

In the remainder of this paper, we illustrate through example the new type of geometric / algebraic reasoning enabled by the software.

\section{Why work symbolically?}

Both generalization and specialization have their place in the toolbox of techniques used in mathematical discovery [4]. A dynamic geometry system lends itself particularly to specialization, in that it displays a specific numeric instance 


$$
\text { "todd" — 2009/2/18 — 23:25 — page 306 — \#4 }
$$

of a general configuration. Dragging allows a user to make a one dimensional traverse through the multi-dimensional space of possible instances and is particularly good at identifying invariance. This is a weak form of generalization. Symbolic measurement from a drawing, however, provides a means of genuine generalization.

To take an example, in Figure 3(a), a pair of circles of unit radius are tangential, and mutually tangent and interior to a circle of radius 2 . Taking as a starting point one of the circles of unit radius, a sequence of circles is defined, each of which is tangential to the previous member of the sequence, to the outer circle of radius 2 , and to the other circle of unit radius. The radii of the circles forms the numerical sequence:

$$
1, \frac{2}{3}, \frac{1}{3}, \frac{2}{11}, \ldots
$$

If we perform the same construction with initial circles of general radius: $r, s$, and $r+s$, (Figure 3(b)) the structure in this generization of the original problem is more readily apparent:

$$
r, \frac{r s(r+s)}{r^{2}+r s+s^{2}}, \frac{r s(r+s)}{4 r^{2}+r s+s^{2}}, \frac{r s(r+s)}{9 r^{2}+r s+s^{2}}
$$

The first element seems out of place, until we notice that:

$$
r=\frac{r s(r+s)}{0 r^{2}+r s+s^{2}}
$$

The same example can be used to illustrate the use of symbolic geometry in proof. Figure $3(\mathrm{~b})$ exhibits a clear pattern, that the $n^{\text {th }}$ circle in our sequence should have radius:

$$
R_{n}=\frac{r s(r+s)}{n^{2} r^{2}+r s+s^{2}}
$$

We can prove this general using induction, and Geometry Expressions as follows.

Figure 3(b) shows that the result holds for $n=1$. For an inductive step, we create a circle with the appropriate tangencies and the radius $\frac{r s(r+s)}{n^{2} r^{2}+r s+s^{2}}$ (Figure 4). We now create a circle tangent to this and the other two circles, and examine its radius. A little algebraic manipulation will put this in the form $\frac{r s(r+s)}{(n+1)^{2} r^{2}+r s+s^{2}}$. 

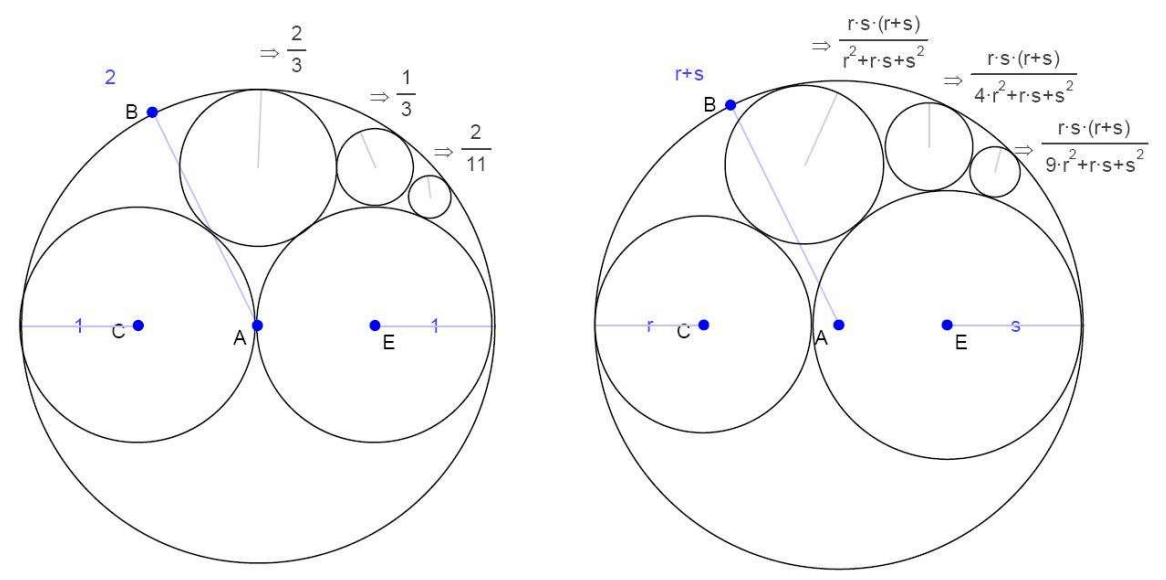

Figure 3. (a) While the pattern of the sequence of radii is not immediately obvious in the numerical instance, (b) the symbolic generalization reveals a clear pattern

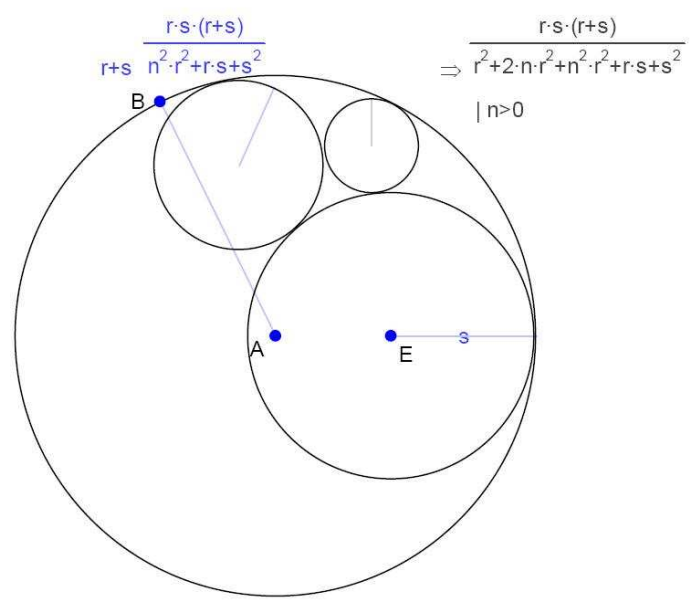

Figure 4. Inductive step in proof of general radius equation (1)

Symbolic Geometry and CAS working hand in hand

Symbolic Geometry working together with CAS facilitates a new paradigm for problem solving and mathematical discovery: brute force application of standard techniques using technology, followed by geometric insight after a solution is obtained, followed finally by the creation of a non-technology based proof. 
As an example, we consider the following problem (Regiomantus' Maximum Problem) [1].

At what point on the earth's surface does a perpendicularly

suspended rod appear longest?

We can approach this initially in a routine fashion. In Figure 5, the earth has radius $r$, and a rod of length $b$ is suspended in space distance $a$ above the surface of the earth. The angle subtended by the rod at point $E$ on the surface of the earth angle $\theta$ from the direction of the rod is calculated by Geometry Expressions:

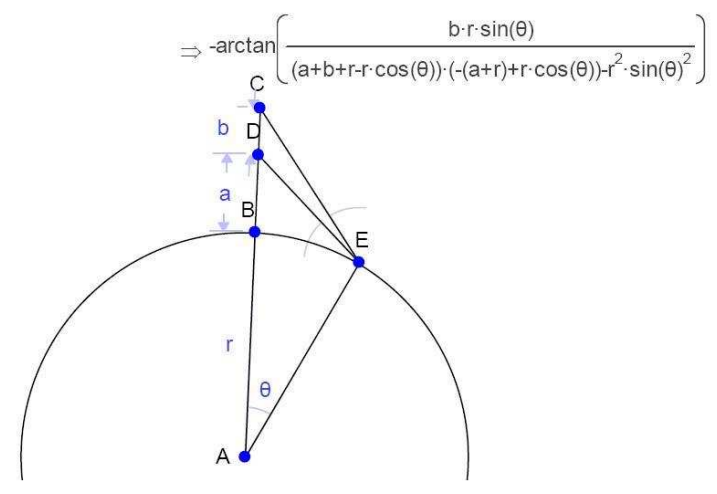

Figure 5. Construction of Regiomontanus' Maximum Problem in Geometry Expressions

Copying into Maple, we can differentiate and solve:

$$
-\arctan \left(\frac{\sin (\theta) r b}{(-a-r+\cos (\theta) r)(a+b+r-\cos (\theta) r)-\sin (\theta)^{2} r^{2}}\right)
$$

$>\operatorname{solve}(\operatorname{diff}(\%$, theta $)=0$, theta $)$;

$$
\begin{gathered}
\arctan \left(\frac{\sqrt{4 a^{2} r^{2}+4 b a r^{2}+2 b^{2} a r+6 a^{2} r b+a^{4}+2 b a^{3}+4 a^{3} r+b^{2} a^{2}}}{a^{2}+b a+2 a r+r b+2 r^{2}},\right. \\
\left.\frac{r(2 a+b+2 r)}{a^{2}+b a+2 a r+r b+2 r^{2}}\right) \\
\arctan \left(-\frac{\sqrt{4 a^{2} r^{2}+4 b a r^{2}+2 b^{2} a r+6 a^{2} r b+a^{4}+2 b a^{3}+4 a^{3} r+b^{2} a^{2}}}{a^{2}+b a+2 a r+r b+2 r^{2}},\right. \\
\left.\frac{r(2 a+b+2 r)}{a^{2}+b a+2 a r+r b+2 r^{2}}\right) .
\end{gathered}
$$




$$
\text { "todd" — 2009/2/18 — 23:25 — page } 309-\# 7
$$

Copying a solution back into Geometry Expressions allows us to visualize it (Figure 6).

Geometric insight can be had by drawing the circumcircle of $C D E$, and observing that it is tangent to $A E$ (Figure 6). As the circumcircle is the locus of all points $F$ such that angle $C F D=\operatorname{angle} C E D$, maximal value for $C E D$ is clearly attained when this locus touches but does not cut the original circle.

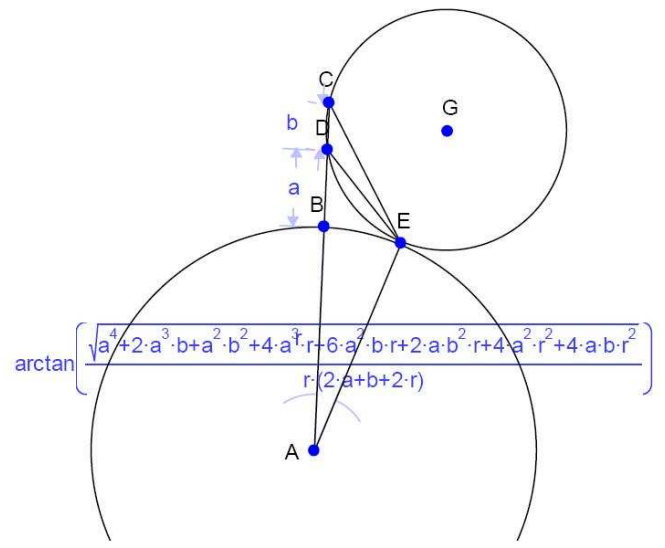

Figure 6. Visualization of the optimal solution to the Regiomontanus' Maximum Problem in Geometry Expressions

\section{Conclusion}

A Symbolic Geometry system such as Geometry Expressions is an effective bridge between the two dominant mathematics education technologies: dynamic geometry and CAS. Symbolic Geometry allows problems to be solved through routine application of standard techniques. The CAS can be relied upon to perform the, perhaps complicated, manipulations required to generate a solution. In short, Geometry Expressions helps with problem formulation, CAS with solution from the formuilation.

For example, while Regiomantus' Maximum Problem (Figure 5) admits an elegant geometric proof with the right insight, it also yields to a straightforward application of calculus. 


$$
\text { "todd" — 2009/2/18 — 23:25 — page } 310-\# 8
$$

Symbolic Geometry software can be seen as providing the missing link between DG and CAS, and in the process substantially extending the geometric discovery learning process into algebra and calculus.

\section{References}

[1] H. Dorrie, "100 Great Problems of Elementary Mathematics", Dover, New York, 1965.

[2] T. Gawlick, On Dynamic Geometry Software in the Regular Classroom, International Reviews on Mathematical Education 34, no. 3 (2002), 85-92.

[3] J. R. King and D. Schattschneider (eds.), Geometry turned on! Dynamic software in learning, teaching, and research, Mathematical Association of America, 1997.

[4] G. Polya, How to Solve it, Princeton University Press, Princeton, NJ., 1945.

PHILIP TODD

SALTIRE SOFTWARE

PO BOX 1565

BEAVERTON OR 97075

USA

E-mail: philt@saltire.com

(Received September, 2007) 